\title{
An Empirical Analysis of the Progress in Wireless Communication Generations
}

\author{
Kevin Luo \\ Kobe University \\ Kobe, Japan \\ kevin.luo@stu.kobe-u.ac.jp
}

\author{
Shuping Dang \\ King Abdullah University of Science \\ and Technology \\ Thuwal, Saudi Arabia \\ shuping.dang@kaust.edu.sa
}

\author{
Chuanting Zhang \\ King Abdullah University of Science \\ and Technology \\ Thuwal, Saudi Arabia \\ chuanting.zhang@kaust.edu.sa
}

\author{
Basem Shihada \\ King Abdullah University of Science \\ and Technology \\ Thuwal, Saudi Arabia \\ basem.shihada@kaust.edu.sa
}

\author{
Mohamed-Slim Alouini \\ King Abdullah University of Science \\ and Technology \\ Thuwal, Saudi Arabia \\ slim.alouini@kaust.edu.sa
}

\begin{abstract}
The controversy and argument on the usefulness of the physical layer (PHY) academic research for wireless communications are long-standing since the cellular communication paradigm gets to its maturity. In particular, researchers suspect that the performance improvement in cellular communications is primarily attributable to the increases in telecommunication infrastructure and radio spectrum instead of the $\mathrm{PHY}$ academic research, whereas concrete evidence is lacking. To respond to this controversy from an objective perspective, we employ econometric approaches to quantify the contributions of the PHY academic research and other performance determinants. Through empirical analysis and the quantitative evidence obtained, albeit preliminary, we shed light on the following issues: 1) what determines the cross-national differences in cellular network performance; 2) to what extent the PHY academic research and other factors affect cellular network performance; 3) what suggestions we can obtain from the data analysis for the stakeholders of the PHY research.
\end{abstract}

\section{CCS CONCEPTS}

- Networks $\rightarrow$ Network performance modeling; Network experimentation.

\section{KEYWORDS}

Physical layer research, cellular communications, empirical analysis, econometrics, $5 \mathrm{G}$ communications.

\section{ACM Reference Format:}

Kevin Luo, Shuping Dang, Chuanting Zhang, Basem Shihada, and MohamedSlim Alouini. 2020. An Empirical Analysis of the Progress in Wireless Communication Generations. In MobiQuitous 2020 - 17th EAI International Conference on Mobile and Ubiquitous Systems: Computing, Networking and Services

Permission to make digital or hard copies of all or part of this work for personal or classroom use is granted without fee provided that copies are not made or distributed for profit or commercial advantage and that copies bear this notice and the full citation on the first page. Copyrights for components of this work owned by others than ACM must be honored. Abstracting with credit is permitted. To copy otherwise, or republish, to post on servers or to redistribute to lists, requires prior specific permission and/or a fee. Request permissions from permissions@acm.org.

MobiQuitous20, December 7-9, 2020, Darmstadt, Germany

(c) 2020 Association for Computing Machinery.

ACM ISBN 978-1-4503-8840-5/20/12 . \$ $\$ 15.00$

https://doi.org/10.1145/3448891.3448894
(MobiQuitous 20), December 7-9, 2020, Darmstadt, Germany. ACM, New York, NY, USA, 10 pages. https://doi.org/10.1145/3448891.3448894

\section{INTRODUCTION}

Telecommunications have experienced a high-speed developing epoch in the past decades, and the constructive influence of this field on human society and technological development can never be overestimated [14]. Among a variety of taxonomic branches of telecommunications, cellular communications have become ubiquitous and increasingly related to the daily life of everyone [26]. Due to the importance of cellular communications, enormous financial resources and human capital have been devoted to academia for scientific research over the past decades [2,4]. As a result of these investments, a number of ground-breaking communication technologies were invented and implemented to significantly ease our daily communications nowadays, including multiple-input and multiple-output (MIMO) system [31], orthogonal frequency-division multiplexing (OFDM) [28], cooperative relaying system [17], software-defined networking (SDN) [16], and artificial intelligence driven wireless communications [19]. As the cellular concept becomes increasingly ripe, there exists another voice questioning the usefulness of the physical layer (PHY) academic research for future wireless communications [4], in particular, the relation between the PHY research activities and service improvements on practical cellular implementations [7].

Before we are going to talk about the specific PHY layer research, it is instructive to make it clear what PHY layer is. The term "physical layer" originated from the Open Systems Interconnection (OSI) model [32], which is a conceptual model that characterizes the functions of a telecommunication system irrespective of its essential structures and implementing technologies. Compared with the conceptual OSI model, the Transmission Control Protocol/Internet Protocol (TCP/IP) model is a simplified version of the OSI model and the practical standards that allow communications over largescale networks. Figure 1 gives a graphical overview of both the OSI model and the TCP/IP model and also introduces related functions of each layer. Both models aim at the standardization of various communication systems with unified communication protocols. PHY lies at the bottom layer of the OSI model and is integrated in the link layer of the TCP/IP model. 


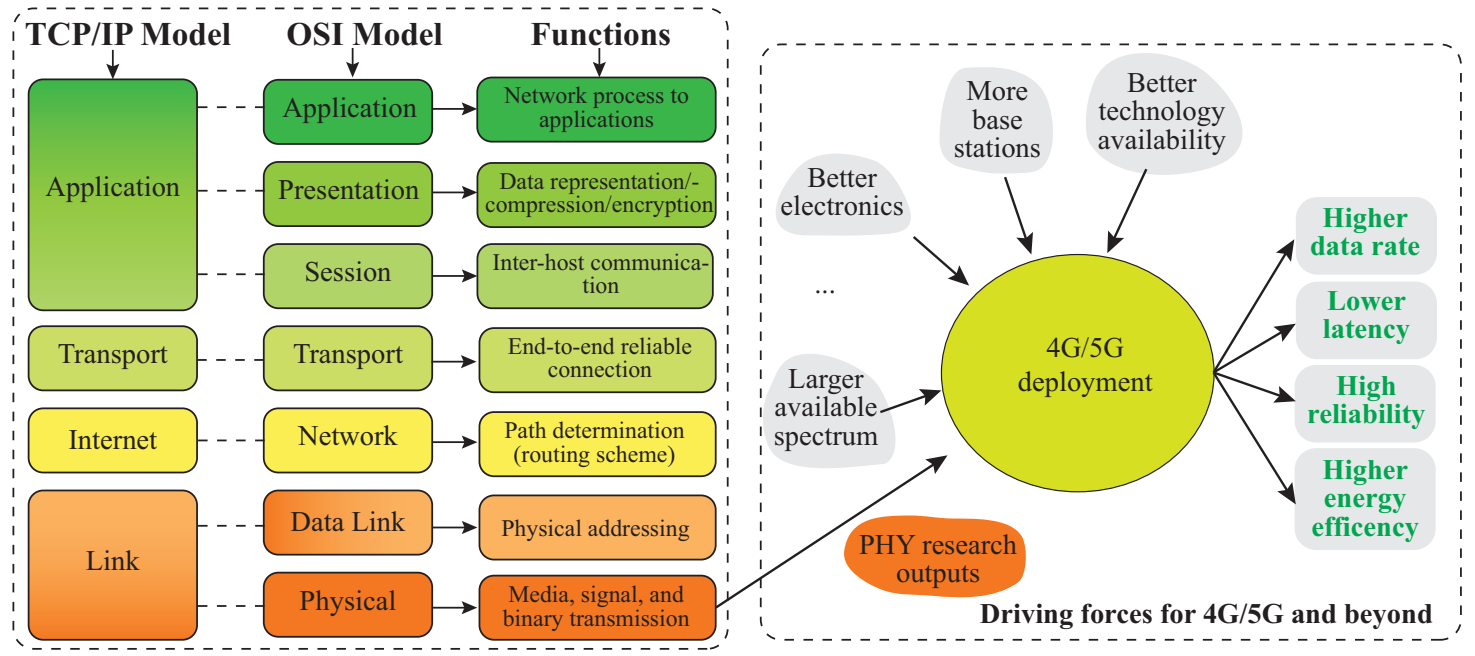

Figure 1: The computer networking models, i.e., TCP/IP model and OSI model, and summary of the 4G/5G development from a systematic viewpoint.

Figure 1 also summarizes the determinants of the $4 \mathrm{G} / 5 \mathrm{G}$ development from a systematic viewpoint, in which the technology evolution is affected by multiple factors and yields many performance advantages. However, the system per se is still a black box to observers, because of the sophisticated and interconnected relationship among all factors. Although technological breakthroughs are of great importance, it is conjectured that the dominant factors for improving practical cellular systems in recent years refer to a higher number of base stations (BSs) and a wider radio spectrum in use $[2,5,7,8]$. The former is a capital-intensive growth pattern, and the latter is more related to electronics and manufacturing industry [20], which might not be directly associated with the PHY research. In this paper, we follow the broad definition of PHY research within the wireless community. The PHY research dedicates to the information transmission and reception mechanisms from transmitters to receivers over communication channels, which also incorporates complex network and system configurations, e.g., multi-user, multi-cell, multi-carrier, and multihop configurations [7]. The two objectives in the PHY research realm can mainly be summarized to be the performance analysis and the performance optimization [27]. Numerical simulations are one of the most crucial tools to verify the correctness and contributions of PHY research outcomes [13].

Together with the ' $5 \mathrm{G}$ enthusiasm' and now even the ' $6 \mathrm{G}$ enthusiasm,' a number of novel PHY communication conceptions, paradigms, and technologies have been proposed and investigated in depth [6]. Are they realistic or nothing but hypes? There are ample examples that a number of heated technologies in the past have finally turned out to be hyped, and wireless communications are not exceptional $[9,10]$. These research topics and activities cost enormous financial and human resources, after all [24]. As a result, the controversy and argument on the usefulness of the PHY academic research are long-standing and constructive [4]. In this light, from the research stakeholders' perspective, it is instinctive to concern the following questions: 1) What are the factors determining the cross-national differences in telecommunication performance? 2) To what extent does the PHY academic research affect the cellular network performance? 3) As an engineering discipline, what can we do to maximize the usefulness of the PHY academic research?

To provide some clues and preliminary answers to these questions in an objective manner, we employ a set of methodologies of econometrics to carry out the analysis. To the best of our knowledge, the study presented in this paper is the first 'empirical telecommunication research' that quantitatively investigates the deductions of communication theory; it is also the first empirical attempt to evaluate the usefulness of the PHY research and quantify the contributions of different determinants to cellular network performance.

\section{RELATED WORK AND CONTRIBUTIONS}

There is an age-old concern of the usefulness of academic research in shaping ripe technological paradigms. Just as the current suspicion of $5 \mathrm{G}$ and $6 \mathrm{G}$, researchers in the $3 \mathrm{G}$ era have already questioned $4 \mathrm{G}$ for whether it has suffered a lot of trendy buzzwords [20]. Meanwhile, it is noticed that the evolution of wireless communications does not fully obey Moore's Law, even though the performance of wireless communication systems is highly related to the processing capability of electronics installed [20]. This is because other nontechnological factors have an equal or even more significant impact on the performance of wireless communication systems, including standardization, legislation, intellectual property protection, legacy deployments, and a series of complex socio-economic and human factors $[15,22,23]$. More importantly, the performance of wireless communication systems is strictly constrained by the basic physical laws of electromagnetic propagation and electronics [29].

It is further pointed out that only a small portion of wireless technologies have finally become commercially viable civil products and appeared on the market [9]. Overall, timing is critical for successful applications of novel wireless technologies. This article also introduces the roles of academia, industry, and government for wireless technology implementation. In addition, the authors 
study the demands, application scenarios, and marketing incentive schemes. More crucial discussions are also provided around the measures for quantifying the usefulness of academic research and funding strategies.

Estimated according to Cooper's Law, the main contributors to capacity are reduced cell size, wider radio spectrum, and better modulation schemes, among which the reduced cell size dominates [5] Following the statement breaking down the million-fold capacity increase over the past 60 years [5], the authors present a critical and detailed retrospect of the progress in $2 \mathrm{G}, 3 \mathrm{G}$, and $4 \mathrm{G}$ [7]. In this article, the life-or-death problem of PHY is investigated. It is stated that the PHY academic research indeed hits the performance wall of the Shannon-Hartley theorem, and any endeavor aiming at increasing capacity would yield marginal returns. The authors suggest that the PHY research should embark on solving realistic problems, instead of creating academic problems. It has also been stressed that more practical considerations have to be taken into the PHY research, so as to be instructive. Furthermore, it is concluded that the PHY academic research is still alive, but morbid. The authors [8] continue to discuss the practical aspects of the $\mathrm{PHY}$ research and wireless communications in the context of $5 \mathrm{G}$ and examine the key performance indicators (KPIs) of cellular communications.

On the other hand, it is emphasized that the PHY academic research is of high importance [2], because we need to understand and model the behavior of high-frequency radio signal as well as to manage cross-cell interference when densifying communication networks in space and frequency spectrum. Also, the crucial role of the PHY research in engineering education and socio-technical milieu of wireless technology cannot be overlooked [27]. Although insightful and thought-provoking, the analyses and discussions of the pros and cons regarding the PHY academic research in all aforementioned articles are mainly given in a subjective manner, lacking rigorous analysis and quantitative evidence.

To obtain quantitative evidence, many researchers have borrowed the analytical frameworks of sociology in studying telecommunication related issues. Employing regression analyses based on empirical data, the authors [12] study the determinants of the diffusion of mobile telecommunication services in the European Union. The authors find that technological breakthroughs, expansion in frequency spectrum, and the increased market competitiveness are essential for the diffusion of $3 \mathrm{G}$ mobile services. The empirical data also demonstrates a simultaneous increase of BSs and bandwidth in the $3 \mathrm{G}$ era and predicts a robust growth of telecommunication infrastructure in the near future.

To address the effective cooperation between academia and industry, the research [4] conducts an empirical analysis to examine the localized interrelations between academic research and entrepreneurial innovation in the wireless sector. The authors find evidence of regional co-localization of scientific and industrial specializations, as well as the positive feedback between academic publications and patent output. However, publications and patents can be flawed measures of innovation output since they do not necessarily imply useful technologies [1].

The authors employ the sociology of translation to document the socio-technical dynamics of $4 \mathrm{G}$ systems in Korea [25]. A set of factors affecting the evolution of $4 \mathrm{G}$ are hereby identified from both supply and demand sides. The authors present a techno-economic analysis for 4G/LTE networks [11]. In particular, the authors evaluate the economic gains of building different types of BSs by using a techno-economic model and give a flow diagram detailing the techno-economic analytical procedure for 4G/LTE cellular systems. A techno-economic analysis framework is employed to evaluate the costs of the deployment of a $4 \mathrm{G}$ relay-assisted OFDM network [18]. All the above studies provide novel analytical frameworks in evaluating the development of wireless technology.

Empirical methods have enriched the analytical tools in the field of wireless communications. However, existing empirical research works regarding wireless communications have two limitations. First, previous empirical attempts are oriented to address only socioeconomic issues in the wireless sector (e.g., the determinants of mobile service diffusion), instead of the research topics in telecommunication science. As a result, there is an urgent need to extend the empirical knowledge of communication theories. Second, the empirical evidence, in particular, quantitative evidence on the status quo of the PHY research remains scarce, and much of the previous evidence was obtained based on anecdotes and questionable observations (e.g., improper metrics for innovative output). Hence, unified empirical frameworks and solid data supports are indispensable for rigorous empirical assessments of the PHY research status.

To overcome the above limitations, the study in this paper pays careful attention to the consistency between theoretical deduction and empirical modeling, the validity of empirical methods, and metric definitions in quantifying the relative importance of the PHY research and other performance determinants.

\section{EMPIRICAL ANALYSIS}

Currently not only openly discussed in the article 'Is the PHY Layer Dead' [7], academia begins to sense the diminishing return and even stagnation in wireless communications after decades of path-breaking advances. There is widespread speculation that the impressive improvements in mobile communication over the past decades were primarily achieved by the network densification and the increase in spectrum resource (a capital-intensive growth pattern), instead of the exponential growth in published papers and information and communications technology (ICT) patents (a technology-intensive growth pattern). So far, quantitative evidence is still lacking, and no consensus has been achieved yet.

As an applied discipline, the validity of the PHY layer research should be judged by its contribution to the cellular network performance. To evaluate practical scenarios in a unified and simple framework, an econometric analysis is a promising option. Econometric analysis is midway path between simulation and control experiment. It is based on real-world settings instead of parameter assumptions, and it derives parameter estimates in a quasiexperimental manner. We begin with the theoretical modeling of network throughput (the key performance indicator of cellular networks nowadays) and then introduce our estimation method and models based on econometrics.

\subsection{Theoretical and Empirical Modeling}

Stemming from the Shannon-Hartley theorem, the cellular concept was proposed to boost network throughput by limiting the 
Table 1: Definitions and sources of the key metrics.

\begin{tabular}{|c|c|c|c|c|c|c|}
\hline Metric & Average & $\begin{array}{l}\text { Standard } \\
\text { derivation }\end{array}$ & $\operatorname{Max}$ & Min & Definition & Source \\
\hline $\begin{array}{l}\text { Mobile download } \\
\text { speed }\end{array}$ & 26.81 & 14.80 & 65.41 & 6.32 & $\begin{array}{l}\text { Average mobile } \\
\text { download speed in } \\
\text { Mbps }\end{array}$ & $\begin{array}{l}\text { Speedtest Global } \\
\text { Index (May 2019) }\end{array}$ \\
\hline Telecom Towers & 0.57 & 0.66 & 5.56 & 0.03 & $\begin{array}{l}\text { Number of installed } \\
\text { telecom towers per } 1000 \\
\text { mobile subscribers }\end{array}$ & $\begin{array}{l}\text { Telecom Network } \\
\text { Report Ed } 4 \text { (2017), } \\
\text { StatPlan Energy } \\
\text { Research }\end{array}$ \\
\hline Bandwidth & 191.11 & 131.29 & 685.00 & 25.00 & $\begin{array}{l}\text { Bandwidth for downlink } \\
\text { cellular transmissions in } \\
\mathrm{MHz}\end{array}$ & $\begin{array}{l}\text { National Frequency } \\
\text { Allocation Table } \\
\text { (2018) }\end{array}$ \\
\hline Telecom Papers & 3.84 & 3.17 & 10.49 & 0.00 & $\begin{array}{l}\text { Logarithm of the } \\
\text { number of telecom } \\
\text { papers since } 1948\end{array}$ & Web of Science \\
\hline $\begin{array}{l}\text { Telecom Papers } \\
\text { (Relative) }\end{array}$ & -7.59 & 1.97 & -3.99 & -12.16 & $\begin{array}{l}\text { Logarithm of the ratio } \\
\text { of Telecom Papers to } \\
\text { GDP (million USD) }\end{array}$ & Web of Science \\
\hline $\begin{array}{l}\text { Telecom } \\
\text { TopPapers }\end{array}$ & 0.80 & 1.36 & 6.91 & 0.00 & $\begin{array}{l}\text { Logarithm of the } \\
\text { number of telecom } \\
\text { papers cited over } 100 \\
\text { times since } 1948\end{array}$ & Web of Science \\
\hline $\begin{array}{l}\text { Telecom } \\
\text { TopPapers } \\
\text { (Relative) }\end{array}$ & -10.65 & 1.27 & -7.20 & -13.96 & $\begin{array}{l}\text { Logarithm of the ratio } \\
\text { of Telecom TopPapers } \\
\text { to GDP (million USD) }\end{array}$ & Web of Science \\
\hline ICT Patents & 3.44 & 3.57 & 12.33 & 0.00 & $\begin{array}{l}\text { Logarithm of the } \\
\text { number of applications } \\
\text { for ICT related patents }\end{array}$ & $\begin{array}{l}\text { The Global IT Report } \\
\text { (2016) }\end{array}$ \\
\hline $\begin{array}{l}\text { ICT Patents } \\
\text { (Relative) }\end{array}$ & -7.84 & 2.49 & -3.24 & -13.70 & $\begin{array}{l}\text { Logarithm of the ratio } \\
\text { of ICT patents to GDP } \\
\text { (million USD) }\end{array}$ & $\begin{array}{l}\text { The Global IT Report } \\
\text { (2016) }\end{array}$ \\
\hline $\begin{array}{l}\text { Technology } \\
\text { Availability }\end{array}$ & 4.78 & 0.92 & 6.60 & 3.00 & $\begin{array}{l}\text { Rating of availability } \\
\text { level of latest } \\
\text { technologies (from } 1 \text { to } \\
\text { 7) }\end{array}$ & $\begin{array}{l}\text { The Global IT Report } \\
\text { (2016) }\end{array}$ \\
\hline
\end{tabular}

Note: 1) The data on mobile subscribers and GDP were obtained from ITU WTID (2018). 2) To maintain tractability and allow consistent analysis over all countries, countries reported having no research output are assigned with the value of one, so that the logarithm of the corresponding value equals zero. 3) The numbers of country-level ICT patent applications in 2012 and 2013 are available in the Global IT Report (2016), but historical data cannot be found. We thereby assume that the cumulative sum of ICT patent applications is ten times of that in 2012-2013. 4) For simplicity, the downlink bandwidth of time division duplexing (TDD) is assumed to be half of the full bandwidth.

service coverage of each BS to a hexagonal cell, so that scarce frequency spectrum can be reused in different cells with a sufficiently large physical separation. As a result, for a given geographic region, network planners can theoretically provide an arbitrarily high throughput by increasing the number of BSs with a smaller transmit power by a proper frequency reuse scheme [21]. Therefore, assuming that only the interference from the first-layer interfering cells is concerned and that all BSs are homogeneous with the same transmit power $P_{t}$, the downlink normalized network throughput per user over a given geographic region can be approximated as

$$
C \approx \frac{K M}{U N} \log _{2}\left(1+\frac{(3 N)^{\alpha / 2}}{6}\right),
$$

where $K$ is the total number of available channels; $N$ is the number of cells that collectively use these $K$ available channels; $U$ is the number of users subscribing downlink data service; $\alpha$ is the path loss exponent, which characterizes the quality of signal propagation environment; $M$ is the total number of BSs over the entire geographic region required to be covered, and the following restriction shall be satisfied in order to provide appropriate QoS: $M>A \rho P_{t}^{-\alpha / 2}$, where $A$ is the area of the given geographic region required to be covered and $\rho$ is a parameter related to the network and system configurations.

Cooper's Law leads to a speculation that the million-fold improvement in data rate over the past 60 years can be primarily 


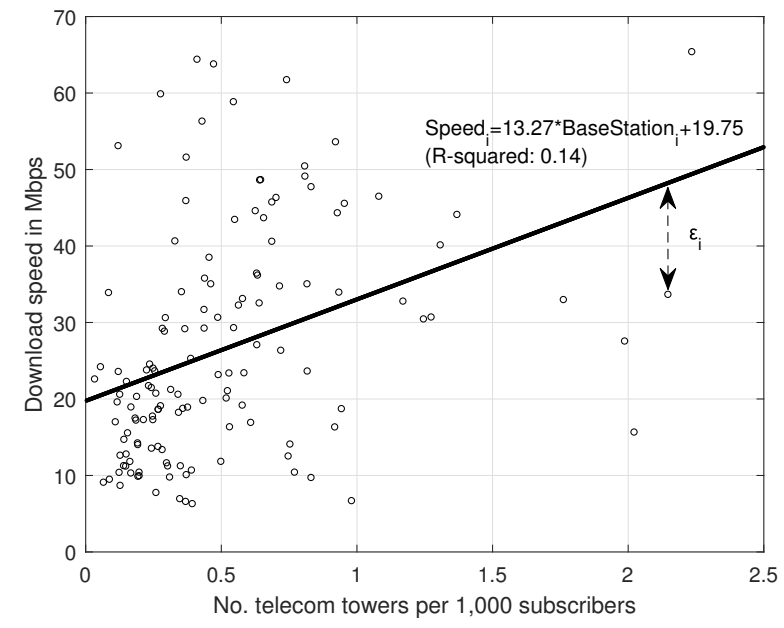

(a)

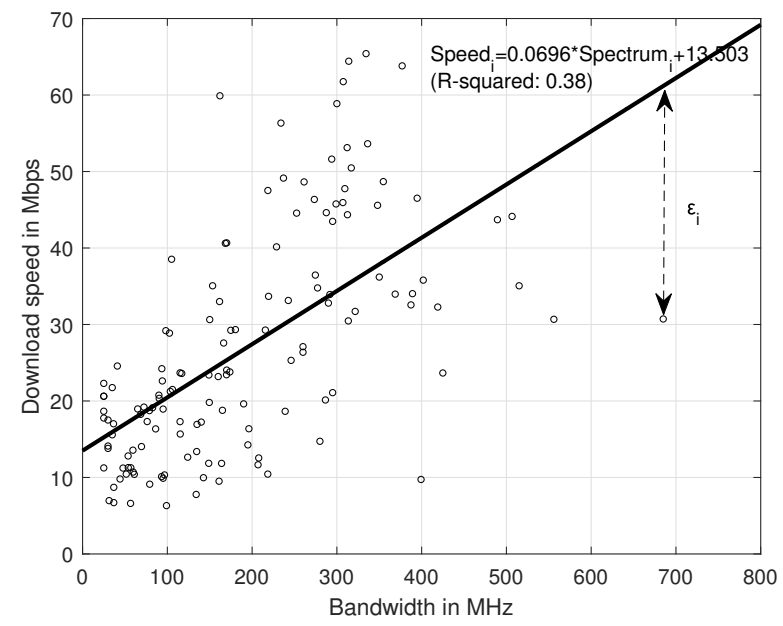

(b)

Figure 2: Two simple linear regression examples: (a) the number of telecommunication towers; (b) bandwidth.

attributed to the densification of BSs (which accounts for a 1600 fold gain) and the expansion of radio spectrum (which accounts for a 30-fold gain), whereas the progress in communication theory has contributed no more than a 10-fold gain [5]. In light of Cooper's Law that the densification of infrastructure and the expansion of radio spectrum serve as the primary engine for network capacity improvement, we construct the econometric model of throughput as

$$
C_{i}=\beta_{1} \frac{K_{i} M_{i}}{U_{i} N_{i}}+\varepsilon_{i}
$$

Consistent with [2], the model in (2) imply that the increases in the BS density and bandwidth have a proportional payoff in terms of throughput, which leads to the benchmark empirical model of throughput as

$$
\begin{aligned}
\text { Speed }_{i}= & \beta_{0}+\beta_{1} \text { BaseStation }_{i} \\
& +\beta_{2} \text { Spectrum }_{i}+\beta_{3} \log \left(\text { Research }_{i}\right)+\delta_{i},
\end{aligned}
$$

where $\beta_{i}$ is the coefficient estimate, which characterizes the impact of the associated factor on the metric; the subscript $i$ is the countryspecific index; Speed $_{i}$ denotes the average mobile download speed in Mbps, which is a perceived KPI of network throughput per user; BaseStation $_{i}$ is the number of installed telecommunication towers per 1000 mobile subscribers, which represents the level of telecommunication infrastructure; Spectrum $_{i}$ is the overall downlink bandwidth in operation; Research ${ }_{i}$ is the cumulative outputs of the PHY research, represented by the numbers of published papers, highly cited papers, or ICT patent applications; $\varepsilon_{i}$ and $\delta_{i}$ represent the error terms.

\subsection{Empirical Analysis and Discussion}

The empirical model given in (3) is a reduced-form linear regression designed to assess the determinants of cross-national differences in the performance of cellular communications. We collect the latest datasets to construct a comprehensive country-level database, which covers 138 nations or regions (observations) and over 100 performance metrics in 2018. Metrics that lead to justifiable and robust empirical regularities are selected as key metrics. For clarity, the definitions and sources of the key metrics are listed in Table 1.

We pay careful attention to the endogeneity and heterogeneity considerations ${ }^{1}$, which are the main challenges confronting econometric applications. To avoid introducing multicollinearity ${ }^{2}$, the estimations examine the proxies for research output separately and incorporate explanatory metrics that have independent implications. We employ the standard ordinary least squares (OLS) method to estimate the unknown parameters in the empirical model shown in (3). Under the standard OLS framework, the principle of least squares chooses the parameters to minimize the sum of the squares of the deviations between actual observations and predicted values [30]:

$$
\begin{aligned}
\hat{\beta}_{j} & =\min _{\beta_{j}}\left\{\sum_{i=1}^{n}\left(y_{i}-\beta_{0}-\beta_{1} x_{i 1}-\cdots-\beta_{j} x_{i j}\right)^{2}\right\} \\
& =\min _{\beta_{j}}\left\{\sum_{i=1}^{n} \varepsilon_{i}^{2}\right\},
\end{aligned}
$$

where $y_{i}$ represents the $i$ th observation (dependent variable), e.g., Speed $_{i}$ in our case; $x_{i j}$ represents the $j$ th explanatory variable (independent variable) for the $i$ th observation; $n$ is the number of observations.

For instance, considering the causal effects of BaseStation $i$ and Spectrum $_{i}$ on Speed ${ }_{i}$, Fig. 2 visualizes the observations from our

\footnotetext{
${ }^{1}$ In econometrics, the endogeneity problem broadly refers to the undesirable correlation between explanatory variables and the error term due to improper model specifications, which violates the exogeneity assumption of the Gauss-Markov theorem and leads to biased parameter estimates. The heterogeneity problem is related to the situation in which the estimated effect differs significantly across different research subjects due to the poor control of individual differences, e.g., ethnicities and cultural backgrounds. ${ }^{2}$ Multicollinearity represents a high degree of linear correlation between explanatory variables due to improper variable settings, which can raise the overall prediction error and invalidate the estimation process.
} 
datasets and the corresponding parameter estimates. In these two simple linear regression examples, the standard OLS method relying on (5) finds the parameters that lead to the minimal prediction error, i.e., $\sum_{i=1}^{n} \varepsilon_{i}^{2}$. Overall, both variables are significantly correlated with the download speed Speed ${ }_{i}$. Relative to BaseStation ${ }_{i}$, Spectrum $_{i}$ has a higher degree of explanatory power (represented by the Rsquared value [30]) and accounts for a greater cross-national variation in Speed $_{i}$. This evidence indicates that Cooper's Law might have overemphasized the relative contribution of BS densification. To improve the overall fitness, the regression model needs to incorporate other performance determinants and take a functional form complying more with the theoretically derived form.

Table 2 displays the OLS estimates for multiple linear regressions, in which the first column is associated with the benchmark estimation, and other columns are related to estimations using alternative sets of metrics. According to the first estimation, mobile download speed is positively and significantly correlated with the number of installed telecommunication towers: each additional telecommunication tower per 1000 subscribers increases the download speed by $4.14 \mathrm{Mbps}$, implying a pronounced effect of telecommunication infrastructure on cellular network performance. Similarly, each 1 $\mathrm{MHz}$ increase in bandwidth leads to a $0.06 \mathrm{Mbps}$ increase in the download speed. Overall, BS density and spectral resource can explain $41.2 \%$ of the cross-national differences in mobile download speed, which validates the explanatory power of the two-factor model but also indicates the existence of other performance determinants.

Based on the first estimation, estimations 2-4 further control for the cross-national differences in the PHY research outputs using different proxies. Overall, estimations using different proxies have reached robust results. As expected, the contribution of highly cited papers is larger than that of less cited papers, although the absolute quantity of highly cited papers is much smaller. The analytical results indicate that the PHY research has statistically significant but only modest effects on the cellular network performance. In particular, the introduction of research outputs leads to only a 4-9\% increase in the explanatory power. Quantitatively, the estimated coefficient suggests that a $1 \%$ increase in the cumulative research outputs contributes to no more than a $0.04 \mathrm{Mbps}$ increase in download speed. In other words, doubling the PHY research outputs will only lead to less than $4 \mathrm{Mbps}$ increase in download speed (note that the estimated coefficient represents the marginal effect). This evidence lends credits to the speculation from Cooper's Law and the widespread concern over the increasing divergence between academia and industry [5, 7].

To approach the theoretical deduction of throughput in (1) and separate the effects of the BS density and spectral resource, we carry out the logarithmic transformation of (2) and obtain

$$
\log \left(C_{i}\right)=\beta_{1} \log \left(\frac{M_{i}}{U_{i} N_{i}}\right)+\beta_{2} \log \left(K_{i}\right)+\varepsilon_{i}
$$

and

$$
\begin{aligned}
\log \left(\text { Speed }_{i}\right)= & \beta_{0}+\beta_{1} \log \left(\text { BaseStation }_{i}\right) \\
& +\beta_{2} \log \left(\text { Spectrum }_{i}\right)+\beta_{3} \log \left(\text { Research }_{i}\right)+\delta_{i},
\end{aligned}
$$

Based on the log-log form, we re-estimate the parameters using the same econometric techniques. The re-estimation examines whether the results are sensitive to the selection of model specifications. More importantly, the re-estimation helps to quantify the performance gains of different inputs and thus makes the estimated coefficients more comparable. In the log-log specification, parameter $\beta_{1}$ represents the elasticity of download speed with respect to the number of BSs, which corresponds to the densification gain and so is the case of bandwidth and the PHY research output. Theoretically, $\beta_{1}$ and $\beta_{2}$ should approach unity, since the performance gains are presumably proportional as suggested by (1). However, empirically, $\beta_{1}$ and $\beta_{2}$ can be less than one due to practical imperfections, but quantitative evidence remains scarce.

Table 3 lists the estimated results under the log-log specification. Overall, the metrics have retained their explanatory power and statistical significance as in the former estimations. Quantitatively, a $1 \%$ increase in the BS density leads to a $0.11-0.13 \%$ increase in mobile download speed; the densification gain of spectral resource is relatively high $(0.23-0.38 \%)$, but also below the theoretical upper bound of unity. On the other hand, again, the contribution of the PHY research is estimated to be statistically significant but only marginal. A $1 \%$ increase in the PHY research output is associated with an increase of no more than $0.11 \%$ in cellular network performance. These findings indicate the possible existence of network resource misallocation, mismatch between research needs and research priority, and large potential for cellular performance improvement via research efforts.

\subsection{Robustness Tests}

Although insightful, the preliminary results in Table 2 and Table 3 could be misleading due to model misspecifications. Perhaps the most central concern is the existence of knowledge diffusion. That is, countries can benefit from technology innovations both within and across borders. Typical cases are the small-size developed countries, such as Luxembourg and the Netherlands, which have advanced mobile networks but rely little on independent research and development. Estimations that overlook cross-national technology absorption will overestimate the statistical significance and contribution of local research outputs.

Another consideration is the issue of spurious correlation. That is, there might be positive correlations between research output, national productivity, and mobile network performance in the statistical sense, although the PHY research does not necessarily contribute to performance improvement. To put it simply, research output can be a redundant by-product in the production process, as indicated by the speculation over the death of the PHY research. Potential candidates are the largest economies, such as the U.S. and Japan, which have superior mobile networks, overcapacity, and substantial research outputs. The above considerations also cast doubts on the validity of cross-national comparisons among absolute levels of research output, since different countries differ considerably in economic scales.

To test these hypotheses, the estimation incorporates the proxy called the availability level of the latest technologies to control for the technology absorptive capacity (TAC) of each country, in contrast to countries' independent research outputs. Moreover, we 
Table 2: Determinants of mobile download speed under the linear specification.

\begin{tabular}{c|c|c|c|c}
\hline \multirow{2}{*}{ Metrics } & \multicolumn{4}{|c}{ Mobile download speed } \\
\cline { 2 - 5 } & 1 & 2 & 3 & 4 \\
\hline Telecom Towers & $4.140^{* * *}(1.26)$ & $4.365^{* * *}(1.20)$ & $4.746^{* * *}(1.20)$ & $4.659^{* * *}(1.06)$ \\
\hline $\begin{array}{c}\text { Bandwidth } \\
\text { Telecom Papers }\end{array}$ & $0.0635^{* * *}(0.01)$ & $0.0434^{* * *}(0.01)$ & $0.0402^{* * *}(0.01)$ & $0.0318^{* *}(0.01)$ \\
\hline Telecom TopPapers & - & $1.248^{* * *}(0.41)$ & - & - \\
\hline ICT Patents & - & - & $3.446^{* * *}(1.25)$ & - \\
\hline Constant & $12.32^{* * *}(1.62)$ & $11.23^{* * *}(1.47)$ & $13.68^{* * *}(1.68)$ & $12.16^{* * *}(1.52)$ \\
\hline Number of observations & 138 & 138 & 138 & 138 \\
\hline R-squared & 0.412 & 0.453 & 0.472 & 0.501 \\
\hline
\end{tabular}

Note: 1) Robust standard errors in parentheses. 2) ${ }^{* * *}: p<0.01 ;{ }^{* *}: p<0.05 ;{ }^{*}: p<0.1$ ( $p$ is the $p$-value in this statistical hypothesis).

Table 3: Determinants of mobile download speed under the $\log -\log$ specification.

\begin{tabular}{c|c|c|c|c}
\hline \multirow{2}{*}{ Metrics } & \multicolumn{4}{|c}{ Mobile download speed } \\
\cline { 2 - 5 } & 1 & 2 & 3 & 4 \\
\hline \hline Telecom Towers & $0.133^{* * *}(0.05)$ & $0.124^{* * *}(0.05)$ & $0.121^{* *}(0.05)$ & $0.110^{* *}(0.05)$ \\
\hline Bandwidth & $0.380^{* * *}(0.06)$ & $0.297^{* * *}(0.07)$ & $0.291^{* * *}(0.06)$ & $0.225^{* * *}(0.06)$ \\
\hline Telecom Papers & - & $0.0354^{* *}(0.02)$ & - & - \\
\hline Telecom TopPapers & - & - & $0.107^{* * *}(0.03)$ & - \\
\hline ICT Patents & - & - & - & $0.0632^{* * *}(0.01)$ \\
\hline Constant & $1.368^{* * *}(0.31)$ & $1.636^{* * *}(0.35)$ & $1.710^{* * *}(0.33)$ & $1.889^{* * *}(0.31)$ \\
\hline Number of observations & 138 & 138 & 138 & 138 \\
\hline R-squared & 0.424 & 0.446 & 0.469 & 0.520 \\
\hline
\end{tabular}

Note: 1) Robust standard errors in parentheses. 2$)^{* * *}: p<0.01 ;{ }^{* *}: p<0.05 ;{ }^{*}: p<0.1$ ( $p$ is the $p$-value in this statistical hypothesis).

construct relative research output as the ratio of research output to gross domestic product (GDP), which measures the strength of research efforts in each country. The transformation from quantity into quality metrics helps to control for economic scales and enable appropriate cross-national comparisons. Table 4 displays the results of the misspecification tests, in which estimations 13 examine the potential overestimation of local research contribution (due to technology absorption) and estimations 4-6 further control for the scale economy effect using relative research outputs.

Through the data presented, mobile network performance relates closely to the TAC: a unit increase in the availability level of the latest technologies leads to a 5.68-7.65 Mbps increase in mobile download speed. As expected, the statistical significance of other determinants reduces as technology availability enters the model, since these metrics simultaneously account for the development level of telecommunication technology, and thus generate multicollinearity. Due to the same reason, estimations 1-3 lead to lower estimates of local research contribution than the previous results. These findings suggest that the model has properly controlled for the effect of technology absorption.
Furthermore, according to estimations 4-6, alternative definitions of research output do not alter the main conclusions drawn by the previous data analysis. These estimations reject the hypothesis of spurious correlation and verify the presence of technology absorption as well as the rate of return on the domestic PHY research. Overall, the empirical analysis points towards robust empirical relationships between mobile download speed, telecommunication infrastructure, spectral resource, and the PHY research. It has been observed that the three-factor empirical model can explain over $50 \%$ of the variations in cellular network performance.

\section{SUGGESTIONS FOR THE STAKEHOLDERS OF THE PHY ACADEMIC RESEARCH}

Similar to the business world, in academia, the criterion of resource allocation is associated with the cost-benefit evaluation of research investment, without which the academic policy-making process can be subjective and counterproductive. On the other hand, the wireless communication community has long sensed the diminishing return of the PHY research [5, 7]. According to our estimates, the marginal return of the PHY research is highly statistically significant but quantitatively small: a $1 \%$ increase in the cumulative research output leads to less than a $0.1 \%$ performance improvement. 
Table 4: Results of model misspecification tests.

\begin{tabular}{|c|c|c|c|c|c|c|}
\hline \multirow{3}{*}{ Metrics } & \multicolumn{6}{|c|}{ Mobile download speed } \\
\hline & \multicolumn{3}{|c|}{ Absolute research output } & \multicolumn{3}{|c|}{ Relative research output } \\
\hline & 1 & 2 & 3 & 4 & 5 & 6 \\
\hline Telecom Towers & $4.379^{* * *}(1.32)$ & $4.559^{* * *}(1.33)$ & $4.448^{* * *}(1.33)$ & $3.522^{* * *}(1.32)$ & $3.689^{* * *}(1.36)$ & $3.847^{* * *}(1.31)$ \\
\hline Bandwidth & $0.0179^{*}(0.01)$ & $0.0191^{* *}(0.01)$ & $0.0201^{* *}(0.01)$ & $0.0163^{*}(0.01)$ & $0.0298^{* * *}(0.01)$ & $0.0199^{* *}(0.01)$ \\
\hline Telecom Papers & $0.742^{* *}(0.35)$ & - & - & $1.726^{* * *}(0.51)$ & - & - \\
\hline Telecom TopPapers & - & $1.809^{* *}(0.83)$ & - & - & $1.427^{* *}(0.68)$ & - \\
\hline ICT Patents & - & - & $0.689^{*}(0.37)$ & - & - & $1.606^{* * *}(0.50)$ \\
\hline Technology Availability & $7.650^{* * *}(1.17)$ & $7.337^{* * *}(1.20)$ & $6.905^{* * *}(1.33)$ & $7.566^{* * *}(1.14)$ & $7.986^{* * *}(1.15)$ & $5.679^{* * *}(1.35)$ \\
\hline Constant & $-18.57^{* * *}(4.80)$ & $-15.97^{* * *}(5.10)$ & $-15.04^{* * *}(5.47)$ & $-1.434(7.17)$ & $-4.044(8.83)$ & $6.211(9.28)$ \\
\hline Number of observations & 138 & 138 & 138 & 136 & 136 & 136 \\
\hline R-squared & 0.586 & 0.587 & 0.584 & 0.600 & 0.580 & 0.598 \\
\hline
\end{tabular}

Note: 1) Robust standard errors in parentheses. 2$)^{* * *}: p<0.01{ }^{* *}: p<0.05 ;{ }^{*}: p<0.1$ ( $p$ is the $p$-value in this statistical hypothesis). 3) For the estimations of relative research output, there are only 136 observations.

Surprisingly, the marginal return of highly cited papers is about three times as that of less cited papers, although the number of highly cited papers is only one-twentieth as that of less cited papers and ICT patents. Accordingly, given that marginal contribution represents the effect of percentage increases in research output, each highly cited paper is equivalent to 60 less cited papers or 30 ICT patents in terms of the payoff of research investment. In this sense, the value of path-breaking research is self-evident.

Today, we need to confront the fact that the impressive performance improvement in the past decades is only marginally attributable to the PHY research. The top priority is not to blame someone for the stasis in the PHY research (indeed, the stasis can be an inevitable feature of disciplines that approach maturity), but to balance the resource allocation towards the sub-disciplines that hold great potentials. With this purpose, we provide a series of suggestions for the stakeholders of the PHY research in the following subsections.

\subsection{Policymakers}

There is no doubt that administrative intervention has a significant impact on applied disciplines, including wireless communications. Policy orientation should focus on not only the prospective wireless technologies that do not have solid foundation nowadays, but also on the practical wireless technologies that can benefit the society in the foreseeable future. Meanwhile, as network speed and throughput can be well satisfied by building more BSs and expanding spectrum (at least for $5 \mathrm{G}$ and $6 \mathrm{G}$ ) [3], the PHY research should be justified by the improvements on other performance evaluation metrics. Consequently, more feedback and needs from the end beneficiaries of the PHY research should be quantified and taken into account. Under such a need-oriented ideology, quality of experience (QoE) would be adopted as a pivotal metric for making policies and industrial plans. For example, in addition to the conventional metrics (e.g., network speed, throughput, and latency), security, secrecy, and privacy can be quantified and adopted as the key features when blueprinting next generation networks. In addition, governmental subsidies should be provided to lead indirectly to global profits in accordance with the Sustainable Development Goals (SDGs) set by the United Nations General Assembly in the 2030 Agenda.

\subsection{Funders}

When assessing a project proposal applying for financial support, funders should pay attention to not only how many papers will be published or how many patents will be applied at the end of the project cycle, but also the possibility and vision of technology transfer as well as profitability. The assessment of potential market value and practicability should be based on the real needs of end users, which is an important reference point of funding applications. It is highly suggested to add these economic features as part of funding strategies and carry out a long-term tracking and evaluation of them. Then, a new evaluation system of the research outcomes could be constructed to appraise how good a project has been from both technological and socio-economic perspectives. Furthermore, funding priority should be given to the projects contributing to the SDGs. In summary, funding systems have to be efficient and instructive, since they serve as the core mechanism in stimulating creative thinking and innovative technologies in the ongoing 5G evolution.

\subsection{Researchers}

To some extent, telecommunications are becoming a subject of esoteric mathematical models and far-fetched theories, which renders the field gradually divorced from reality. Many communication theories are barely understandable to telecommunication practitioners, and their applicability has not been proved yet. Consequently, new research directions are the key to restore the discipline to its former 
vitality. The real problem is not why we have devoted so much to the PHY research, but why we have so far de-emphasized the nonPHY research and the needs from end beneficiaries. Accordingly, the wireless research community needs to pay more attention to alternative approaches to the fundamental questions of telecommunication science. End beneficiaries should also have a voice in shaping research agendas. Researchers with different backgrounds should also be encouraged to address the same problems using multidisciplinary approaches, such as the empirical methods that can serve as a bridge between academic and real-world problems. Researchers might also borrow the PESTEL analysis (PESTEL: an acronym that stands for Political, Economic, Social, Technological, Environmental and Legal factors) from business studies to appraise the prospect of wireless technology, instead of simply focusing on the academic scholarship and/or the profitability of mobile network operators.

\section{CLOSING REMARKS AND FUTURE WORK}

In this paper, we employed econometric methodologies to investigate the usefulness of the PHY academic research and compared it with other non-technological factors in terms of their contribution to the cellular network performance. The empirical analysis confirmed that the main contributor to the service improvement is the densification of cellular network, whereas only a marginal portion of the service improvement can be attributed to the PHY academic research. The empirical evidence also suggests that highly cited publications have much larger and more statistically significant impacts on cellular network performance than other research outputs. Overall, the analysis has documented robust empirical relations between network capacity, BS density, spectral resource, and the PHY research activities. Based on these findings, we provided a series of suggestions for the stakeholders of the PHY academic research.

Turn to the core issue under discussion: is the PHY research dead? In conclusion, NO, the PHY research is not dead yet, as the marginal return of the PHY research remains statistically significant, and there is indeed a huge potential for performance improvements via research efforts (as suggested by the low densification gains of telecommunication infrastructure and spectral resource). However, we also need to confront and react to the diminishing return of the PHY research. Close collaboration between policymakers, funders, and researchers is indispensable to restore the quondam vitality of the PHY research. Through the results and discussions presented in this paper, it is reasonable to state that the PHY research is sick.

Employing empirical analysis based on actual observations, researchers are able to answer real-world problems such as the relative importance of academic research, other than being confined to the mathematical problems of interest only to academics. This study explores a new angle to quantify the marginal contributions of BS density, spectral resource, and research output in promoting telecommunication performance. This study is helpful to reconcile the controversy on the usefulness of the PHY research.

However, it is important to stress that our analysis remains preliminary and tentative. The empirical analysis has focused on cellular communications, which left other branches of wireless communications unexplained (e.g., machine-to-machine (M2M) communications and satellite communications). In addition, the absence of reliable data has discouraged the research efforts into important topics, such as the impacts of intellectual property protection, technological standardization, and national innovation strategy on the cellular network performance. Also, due to the data insufficiency, our attempts are limited to simple empirical regularities, other than rigorous causal relations between research investment and performance improvement. All in all, as we are entering the era of 'Big Data,' the PHY research should center more on the exploitation of statistical systems, which will enrich the analytical tools to explore promising research directions hidden in telecommunication science.

\section{REFERENCES}

[1] Zoltan J Acs, David B Audretsch, and Maryann P Feldman. 1992. Real effects of academic research: Comment. The American Economic Review 82, 1 (1992), 363-367.

[2] J. G. Andrews, S. Buzzi, W. Choi, S. V. Hanly, A. Lozano, A. C. K. Soong, and J. C. Zhang. 2014. What Will 5G Be? IEEE fournal on Selected Areas in Communications 32, 6 (June 2014), 1065-1082. https://doi.org/10.1109/JSAC.2014.2328098

[3] N. Bhushan, J. Li, D. Malladi, R. Gilmore, D. Brenner, A. Damnjanovic, R. T. Sukhavasi, C. Patel, and S. Geirhofer. 2014. Network densification: The dominant theme for wireless evolution into 5G. IEEE Communications Magazine 52, 2 (Feb. 2014), 82-89. https://doi.org/10.1109/MCOM.2014.6736747

[4] Mario Calderini and Giuseppe Scellato. 2005. Academic research, technological specialization and the innovation performance in European regions: An empirical analysis in the wireless sector. Industrial and Corporate Change 14, 2 (Mar. 2005), 279-305. https://doi.org/10.1093/icc/dth054

[5] V. Chandrasekhar, J. G. Andrews, and A. Gatherer. 2008. Femtocell networks: A survey. IEEE Commun. Mag. 46, 9 (Sept. 2008), 59-67. https://doi.org/10.1109/ MCOM.2008.4623708

[6] Shuping Dang, Osama Amin, Basem Shihada, and Mohamed-Slim Alouini. 2020. What should 6G be? Nature Electronics 3, 1 (2020), 20-29.

[7] M. Dohler, R. W. Heath, A. Lozano, C. B. Papadias, and R. A. Valenzuela. 2011. Is the PHY layer dead? IEEE Communications Magazine 49, 4 (Apr. 2011), 159-165. https://doi.org/10.1109/MCOM.2011.5741160

[8] M. Dohler, T. Mahmoodi, M. A. Lema, and M. Condoluci. 2017. Future of mobile. In Proc. IEEE EuCNC. 1-5. https://doi.org/10.1109/EuCNC.2017.7980729

[9] M. Dohler, D. Meddour, S. Senouci, and A. Saadani. 2008. Cooperation in 4G - Hype or Ripe? IEEE Tech. and Soc. Mag. 27, 1 (Spring 2008), 13-17. https: //doi.org/10.1109/MTS.2008.918035

[10] Stephen Fox. 2018. Irresponsible Research and Innovation? Applying Findings from Neuroscience to Analysis of Unsustainable Hype Cycles. Sustainability 10, 10 (2018). https://doi.org/10.3390/su10103472

[11] Zoraida Frias and Jorge Pérez. 2012. Techno-economic analysis of femtocell deployment in long-term evolution networks. EURASIP fournal on Wireless Communications and Networking 2012, 1 (18 Sept 2012), 288. https://doi.org/10. 1186/1687-1499-2012-288

[12] Harald Gruber and Frank Verboven. 2001. The diffusion of mobile telecommunications services in the European Union. European Economic Review 45, 3 (2001), 577-588.

[13] Michel C Jeruchim, Philip Balaban, and K Sam Shanmugan. 2006. Simulation of communication systems: modeling, methodology and techniques. Springer Science $\&$ Business Media.

[14] Ze-Min Jiang. 2009. Development of IT industry in China in the new age. fournal of Shanghai fiaotong University (Science) 14, 1 (2009), 1-24.

[15] Kalevi Kilkki. 2008. Quality of experience in communications ecosystem. fournal of Universal Computer Science 14, 5 (2008), 615-624.

[16] D. Kreutz, F. M. V. Ramos, P. E. Veríssimo, C. E. Rothenberg, S. Azodolmolky, and S. Uhlig. 2015. Software-Defined Networking: A Comprehensive Survey. Proc. IEEE 103, 1 (Jan. 2015), 14-76. https://doi.org/10.1109/JPROC.2014.2371999

[17] J. N. Laneman, D. N. C. Tse, and G. W. Wornell. 2004. Cooperative diversity in wireless networks: Efficient protocols and outage behavior. IEEE Transactions on Information Theory 50, 12 (Dec. 2004), 3062-3080. https://doi.org/10.1109/TIT. 2004.838089

[18] Antolín Moral, Josep Vidal, Jorge Pérez, Adrián Agustín, Ninoslav Marina, and Anders Høst-Madsen. 2011. Technoeconomic evaluation of cooperative relaying transmission techniques in OFDM cellular networks. EURASIP fournal on Advances in Signal Processing 2011 (2011). 
[19] T. O'Shea and J. Hoydis. 2017. An Introduction to Deep Learning for the Physical Layer. IEEE Transactions on Cognitive Communications and Networking 3, 4 (Dec. 2017), 563-575. https://doi.org/10.1109/TCCN.2017.2758370

[20] Y. Raivio. 2001. 4G-Hype or reality. In Proc. International Conference on $3 G$ Mobile Communication Technologies. London, UK, 346-350. https://doi.org/10.1049/cp: 20010069

[21] Theodore S. Rappaport. 2010. Wireless Communications: Principles And Practice. Pearson Education.

[22] K. U. Rehman Laghari and K. Connelly. 2012. Toward total quality of experience A QoE model in a communication ecosystem. IEEE Communications Magazine 50, 4 (Apr. 2012), 58-65. https://doi.org/10.1109/MCOM.2012.6178834

[23] Peter Reichl. 2010. From Charging for Quality of Service to Charging for Quality of Experience. Annals of Telecommunications-annales des télécommunications 65 3-4 (2010), 189-199.

[24] R. Saracco. 2003. Forecasting the future of information technology: how to make research investment more cost-effective? IEEE Communications Magazine 41, 12 (Dec. 2003), 38-45. https://doi.org/10.1109/MCOM.2003.1252797

[25] Dong-Hee Shin, Hyunseung Choo, and Khisu Beom. 2011. Socio-technical dynamics in the development of next generation mobile network: Translation beyond 3G. Technological Forecasting and Social Change 78, 3 (2011), 514-525.

[26] C. Wang, F. Haider, X. Gao, X. You, Y. Yang, D. Yuan, H. M. Aggoune, H. Haas, S. Fletcher, and E. Hepsaydir. 2014. Cellular architecture and key technologies for $5 \mathrm{G}$ wireless communication networks. IEEE Communications Magazine 52, 2 (Feb. 2014), 122-130. https://doi.org/10.1109/MCOM.2014.6736752

[27] Z. Wang, S. Dang, S. Shaham, Z. Zhang, and Z. Lv. 2019. Basic Research Methodology in Wireless Communications: The First Course for Research-Based Graduate Students. IEEE Access 7 (2019), 86678-86696. https://doi.org/10.1109/ACCESS. 2019.2925708

[28] S. B. Weinstein. 2009. The history of orthogonal frequency-division multiplexing. IEEE Communications Magazine 47, 11 (Nov. 2009), 26-35. https://doi.org/10. 1109/MCOM.2009.5307460

[29] G. Wiechman and I. Sason. 2008. An Improved Sphere-Packing Bound for FiniteLength Codes Over Symmetric Memoryless Channels. IEEE Transactions on Information Theory 54, 5 (May 2008), 1962-1990. https://doi.org/10.1109/TIT. 2008.920216

[30] Jeffrey M Wooldridge. 2016. Introductory Econometrics: A Modern Approach. Nelson Education.

[31] Lizhong Zheng and D. N. C. Tse. 2003. Diversity and multiplexing: A fundamental tradeoff in multiple-antenna channels. IEEE Transactions on Information Theory 49, 5 (May 2003), 1073-1096. https://doi.org/10.1109/TIT.2003.810646

[32] H. Zimmermann. 1980. OSI Reference Model - The ISO Model of Architecture for Open Systems Interconnection. IEEE Transactions on Communications 28, 4 (April 1980), 425-432. https://doi.org/10.1109/TCOM.1980.1094702 